\title{
Follow-Up Liver Stiffness Measurements after Liver Resection Influence Oncologic Outcomes of Hepatitis-B-Associated Hepatocellular Carcinoma with Liver Cirrhosis
}

\author{
Jung Il Lee ${ }^{1,2, * \mathbb{D}}$, Hyun Woong Lee ${ }^{1,2}$, Seung Up Kim ${ }^{1,3} \mathbb{D}$, Sang Hoon Ahn ${ }^{1,3}$ \\ and Kwan Sik Lee ${ }^{1,2}$ \\ 1 Department of Internal Medicine, Yonsei University College of Medicine, Seoul 03722, Korea; \\ LHWDOC@yuhs.ac (H.W.L.); KSUKOREA@yuhs.ac (S.U.K.); AHNSH@yuhs.ac (S.H.A.); \\ LEEKS519@yuhs.ac (K.S.L.) \\ 2 Gangnam Severance Hospital, Yonsei University College of Medicine, Seoul 06273, Korea \\ 3 Severance Hospital, Yonsei University College of Medicine, Seoul 03722, Korea \\ * Correspondence: mdflorence@yuhs.ac; Tel.: +82-2-2019-4365; Fax: +82-2-3463-3882
}

Received: 14 February 2019; Accepted: 20 March 2019; Published: 25 March 2019

\begin{abstract}
The severity of liver fibrosis can be noninvasively evaluated by measuring liver stiffness (LS) using transient elastography. This study aimed to evaluate the prognostic value of achieving low liver stiffness measurement (LSM) in patients with cirrhosis confirmed from the resected liver due to hepatocellular carcinoma (HCC). A total of 184 patients that received curative surgery for HCC related to the hepatitis B virus at Barcelona Clinic Liver Cancer stage 0-A, and had a METAVIR fibrosis score of 4 were investigated. LSM significantly decreased after antiviral therapy during follow-up $(p=0.001)$, and achieving LSM $\leq 8$ kilopascal $(\mathrm{kPa})$ suggested a reduced risk of late recurrence (>12 months) (hazard ratio (HR), 0.519; 95\% confidence interval (CI), 0.307-0.877; $p=0.014$ ). Older age at surgery ( $\geq 45$ years) and multiple HCC nodules predicted an increased risk of late recurrence (HR, 3.270; 95\% CI, 1.296-8.251; $p=0.012$; and HR, 3.146; 95\% CI, 1.396-7.089; $p=0.006$ ). Decreased LSM also suggested decreased mortality (HR, 0.251; 95\% CI, 0.086-0.756; $p=0.045$ ) along with baseline low aspartate aminotransferase-to-platelet ratio index (APRI) score $(<1.5)(\mathrm{HR}, 0.251 ; 95 \%$ CI, $0.086-0.759 ; p=0.041)$. Having early HCC recurrence (HR, 9.416; 95\% CI, 3.566-24.861; $p<0.001)$ and microvascular tumor invasion (HR, 3.191; 95\% CI, 1.188-8.568; $p=0.021)$ predicted increased mortality. Among HCC patients with liver cirrhosis under antiviral therapy, achieving low LSM $(\leq 8 \mathrm{kPa}$ ) predicted reduced late HCC recurrence.
\end{abstract}

Keywords: hepatocellular carcinoma; liver cirrhosis; liver stiffness; dynamic changes; antiviral therapy; late recurrence

\section{Introduction}

Liver cirrhosis has been identified as the single most important factor for hepatocellular carcinoma (HCC) development [1]. It is also one of the most important prognostic factors after curative HCC treatment [2,3]. Meanwhile, studies demonstrate that prolonged antiviral therapy is associated with improvement in liver histology and reversal of cirrhosis in chronic infection with hepatitis B virus (HBV) and this might significantly change the prognosis of patients with HBV-related cirrhosis [4-7].

Although liver biopsy is considered the gold standard to diagnose the severity of fibrosis [8], it is an invasive procedure with a possible sampling error, which makes it almost impossible and unethical to perform sequentially. Instead, transient liver stiffness measurement (LSM), obtained by 
transient elastograpy (TE), is a noninvasive means of assessing liver fibrosis documented to be well correlated with biopsy-detected severity of fibrosis [9-11]. Studies reported that TE might be useful in not only detecting advanced liver fibrosis but also in stratifying the risk of HCC development in HBV patients [12-14]. It has been suggested that HBV patients with higher liver stiffness (LS) values (>8 kilopascal $(\mathrm{kPa})$ ) were at a significantly greater risk of HCC development [12]. However, dynamic changes in LSM can be achieved with antiviral therapy [15-17], and it has been recently reported that having LSM $<11.6 \mathrm{kPa}$ after antiviral therapy was associated with a reduced risk of de novo HCC occurrence [18]. Although a decrease in LS values might be associated with decreased incidence of HCC, it has not been well documented whether antiviral therapy and reduction of LSM would have a beneficial effect on oncologic outcomes of HCC patients experiencing cancer development in a cirrhotic background. A study reported that background liver cirrhosis demonstrated a higher incidence of de novo recurrence of HCC [19], and it would be interesting to evaluate whether a reduction in LSM achieved with antiviral therapy would result in favorable prognosis in these patients.

It is not unusual to have LSM decrease as low as the point where advanced liver fibrosis is unlikely to be suspected after prolonged antiviral therapy in patients with clinically diagnosed liver cirrhosis $[20,21]$. However, a reduction in LS values may not necessarily indicate pathologic improvement [22], and the patients may still be at greater risk of HCC carcinogenesis due to the background liver cirrhosis.

Thus, this study evaluated impact of LS reduction to the point where advanced liver fibrosis was unlikely to be suspected using LSM criteria (LS $\leq 8 \mathrm{kpa}$ ), in HBV-related HCC patients whose pathological assessment at the time of HCC occurrence showed advanced liver fibrosis.

\section{Patients and Methods}

\subsection{Patient Enrollment}

This retrospective cohort study was conducted at a tertiary referral hospital in Seoul, Republic of Korea. A total of 1113 patients who underwent liver resection due to HBV-related HCC between January, 2007 and December, 2016 were screened for possible enrollment in this study. Among them, patients that met the following inclusion criteria were selected: curatively resected HBV-related HCC at Barcelona Clinic Liver Cancer (BCLC) stage 0-A; receiving antiviral therapy against HBV started at or after HCC resection for more than 12 months; available LSM, assessed at least 12 months after starting the antiviral therapy; METAVIR fibrosis score of 4, assessed from the resected liver. Exclusion criteria were as follows: receiving antiviral therapy started before the development of HCC; survival time of less than 4 weeks after the liver resection; co-infection with HIV or hepatitis C; previous history of liver transplantation. Finally, 184 patients were recruited for the final analysis. This study was performed in accordance with ethical guidelines of the 1975 Declaration of Helsinki and was approved by the Institutional Review Board of Gangnam Severance Hospital (Permit No: 3-2018-0198).

\subsection{Baseline Workup and Follow-Up}

The index date for study entry was defined as the time of liver resection. The studies performed within 3 months before the surgery were included in the baseline workup. Variables pertaining to HCC including tumor size, number, microvascular invasion, Edmonson grade, and METAVIR fibrosis score were obtained by pathologic evaluation of the resected liver. The patients were regularly followed-up. Liver function tests and alpha-fetoprotein (AFP) measurements were done every 3 months. Dynamic CT or dynamic MRI were regularly performed at intervals no longer than 6 months. As the inclusion criteria indicated, all the patients had LS measured more than once at least 12 months after staring the antiviral therapy. For those with multiple measurements, the data from the last study were considered as the follow-up results. Other biochemical measurements within 1 month before or after the last LS assessment were taken as the follow-up findings. All the patients received the antiviral therapy against HBV starting at or after the surgery for more than 12 months. HCC recurrence was classified as early 
( $\leq 12$ months) or late ( $>12$ months) [23,24]. All the patients were followed until the time of death or for at least 12 months.

\subsection{Assessment of Fibrotic Burden}

All the patients were confirmed to have liver cirrhosis (METAVIR fibrosis score of 4) by pathologic assessment at the time of surgery. In order to determine changes in fibrotic burden by noninvasive means, serum biomarkers of liver fibrosis and LSM were used.

Serum biochemical markers of fibrosis, namely aspartate aminotransferase (AST)-to-platelet ratio index (APRI) [25], and Fibrosis (FIB)-4 [26], were calculated according to published formulae as follows.

$$
\text { APRI }=\left((\text { AST } / \text { upper limit of normal }) / \text { platelet count }\left(10^{9} / \mathrm{L}\right)\right) \times 100
$$

A cutoff value of APRI $\geq 1.5$ was applied to detect a high probability of advanced fibrosis, as previously published [25].

FIB-4 $=($ Age $($ years $) \times$ AST $(\mathrm{U} / \mathrm{L})) /\left(\right.$ platelet count $\left.\left(10^{9} / \mathrm{L}\right) \times(\text { alanine aminotransferase }(\text { ALT })(\mathrm{U} / \mathrm{L}))^{1 / 2}\right)$

A cutoff value of FIB- $4 \geq 2.67$ was used to detect intermediate and high probability of advanced fibrosis [26].

LSM was measured using transient elastography (TE) (FibroScan, EchoSens, Paris, France). Only LSM with at least 10 valid measurements, a success rate of at least $60 \%$, and an interquartile range-to-median ratio $<30 \%$ were considered reliable, following suggestions from previous studies [12, 27]. In this study, $\mathrm{LSM} \leq 8 \mathrm{kPa}$ was considered as low fibrotic burden according to a previous study [12].

\subsection{Statistical Analyses}

Data were summarized as mean \pm standard deviation (SD), median with range, or $n(\%)$, as appropriate. Categorical variables were compared using two-sided $\chi^{2}$-test (or Fisher's exact test, or McNemar test, where appropriate) and continuous variables were compared using independent or paired sample $t$-tests (or Mann-Whitney test, where appropriate). Cumulative HCC recurrence rates and mortality rates were analyzed using Kaplan-Meier's method and compared with the log-rank test. To identify independent risk factors of mortality and HCC recurrence, univariate and subsequent multivariate regression analyses were used. Hazard ratios (HRs) and corresponding $95 \%$ confidence intervals (CIs) were used where indicated.

All statistical analyses were performed using IBM SPSS (version 23). A $p$-value $<0.05$ was considered to indicate statistical significance.

\section{Results}

\subsection{Patient Selection and Demographic Findings}

A total of 184 patients that received curative liver resection for HBV-related HCC at BCLC stage $0-\mathrm{A}$ were investigated. The baseline demographic findings are described in Table 1 according to the HCC recurrence status classified into early ( $\leq 12$ months) or late ( $>12$ months) [23,24]. All the patients were followed until the time of death or for at least 12 months.

The patients that received low-barrier drugs (lamivudine, telbivudine, adefovir) eventually had the medication changed to high-barrier drugs (entecavir, tenofovir). None of the patients experienced biochemical breakthrough due to emergence of antiviral resistant mutation. 
Table 1. Demographic findings of HBV-related HCC patients with cirrhosis treated with liver resection and put under antiviral therapy.

\begin{tabular}{|c|c|c|c|c|}
\hline & $\begin{array}{l}\text { No Recurrence } \\
\quad(n=113)\end{array}$ & $\begin{array}{l}\text { Early Recurrence } \\
\qquad(n=13)\end{array}$ & $\begin{array}{l}\text { Late Recurrence } \\
\qquad(n=58)\end{array}$ & $p$-Value \\
\hline Age, mean \pm SD (years) & $50.4 \pm 9.3$ & $54.2 \pm 7.3$ & $55.6 \pm 8.4$ & $0.003 *$ \\
\hline Male gender & $87(77.0 \%)$ & $11(84.6 \%)$ & $49(84.5 \%)$ & 0.465 \\
\hline $\begin{array}{l}\text { Operation type } \\
\text { Wedge resection } \\
\text { Segmentectomy } \\
\text { Lobectomy }\end{array}$ & $\begin{array}{l}21(18.6 \%) \\
45(39.8 \%) \\
47(41.6 \%) \\
\end{array}$ & $\begin{array}{l}5(38.5 \%) \\
5(38.5 \%) \\
3(23.1 \%)\end{array}$ & $\begin{array}{l}11(19.0 \%) \\
28(48.3 \%) \\
19(32.8 \%)\end{array}$ & 0.329 \\
\hline $\begin{array}{l}\text { Tumor size } \\
\leq 3 \mathrm{~cm} \\
>3 \mathrm{~cm}\end{array}$ & $\begin{array}{l}71(62.8 \%) \\
42(37.2 \%)\end{array}$ & $\begin{array}{l}8(61.5 \%) \\
5(38.5 \%)\end{array}$ & $\begin{array}{l}32(55.2 \%) \\
26(44.8 \%)\end{array}$ & 0.622 \\
\hline $\begin{array}{l}\text { Tumor number } \\
\text { Single } \\
\text { Multiple }\end{array}$ & $\begin{array}{c}110(97.3 \%) \\
3(2.7 \%)\end{array}$ & $\begin{array}{c}10(76.9 \%) \\
3(23.1 \%)\end{array}$ & $\begin{array}{c}51(87.9 \%) \\
7(12.1 \%)\end{array}$ & $0.005 *$ \\
\hline Microvascular invasion & $42(37.2 \%)$ & $6(46.2 \%)$ & $19(32.8 \%)$ & 0.639 \\
\hline $\begin{array}{c}\text { Edmonson grade } \\
1 \\
2 \\
3 \\
4\end{array}$ & $\begin{array}{c}7(6.2 \%) \\
43(38.1 \%) \\
53(46.9 \%) \\
10(8.8 \%)\end{array}$ & $\begin{array}{l}1(7.7 \%) \\
6(46.2 \%) \\
5(38.5 \%) \\
1(7.7 \%)\end{array}$ & $\begin{array}{c}2(3.4 \%) \\
26(44.8 \%) \\
23(39.7 \%) \\
7(12.1 \%)\end{array}$ & 0.899 \\
\hline $\begin{array}{l}\text { AFP, baseline } \\
<200 \mathrm{IU} / \mathrm{mL} \\
\geq 200 \mathrm{IU} / \mathrm{mL}\end{array}$ & $\begin{array}{l}81(71.7 \%) \\
32(28.3 \%)\end{array}$ & $\begin{array}{c}10(76.9 \%) \\
3(23.1 \%)\end{array}$ & $\begin{array}{c}50(86.2 \%) \\
8(13.8 \%)\end{array}$ & 0.105 \\
\hline $\begin{array}{c}\text { MELD score, baseline, mean } \\
\quad \pm \mathrm{SD}\end{array}$ & $16.2 \pm 2.0$ & $16.4 \pm 1.6$ & $16.6 \pm 2.0$ & 0.500 \\
\hline $\begin{array}{l}\text { MELD score, follow-up, } \\
\text { mean } \pm S D\end{array}$ & $16.0 \pm 1.9$ & $16.3 \pm 1.8$ & $16.1 \pm 2.0$ & 0.844 \\
\hline $\begin{array}{c}\text { HBV DNA } \\
<2000 \mathrm{IU} / \mathrm{mL} \\
\geq 2000 \mathrm{IU} / \mathrm{mL}\end{array}$ & $\begin{array}{l}59(52.2 \%) \\
54(47.8 \%)\end{array}$ & $\begin{array}{l}5(38.5 \%) \\
8(61.5 \%)\end{array}$ & $\begin{array}{l}21(36.2 \%) \\
37(63.8 \%)\end{array}$ & 0.117 \\
\hline $\begin{aligned} & \text { HBV DNA } \\
&< 2000 \mathrm{IU} / \mathrm{mL} \\
& \geq 2000 \mathrm{IU} / \mathrm{mL} \\
&\end{aligned}$ & $\begin{array}{c}113(100 \%) \\
0(0 \%)\end{array}$ & $\begin{array}{c}13(100 \%) \\
0(0 \%)\end{array}$ & $\begin{array}{c}57(98.3 \%) \\
1(1.7 \%)\end{array}$ & 0.353 \\
\hline HBeAg positivity & $26(23.0 \%)$ & $5(38.5 \%)$ & $15(25.9 \%)$ & 0.468 \\
\hline $\begin{array}{c}\text { Mode of antiviral therapy } \\
\text { Low barrier }{ }^{\dagger} \\
\text { High barrier }{ }^{\ddagger}\end{array}$ & $\begin{array}{l}36(31.9 \%) \\
77(68.1 \%)\end{array}$ & $\begin{array}{l}5(38.5 \%) \\
8(61.5 \%)\end{array}$ & $\begin{array}{l}28(48.3 \%) \\
30(51.7 \%)\end{array}$ & 0.110 \\
\hline $\begin{array}{l}\text { Duration of antiviral therapy, } \\
\text { mean } \pm \text { SD (months) }\end{array}$ & $71.8 \pm 38.2$ & $51.3 \pm 27.7$ & $85.3 \pm 31.1$ & $0.004 *$ \\
\hline $\begin{array}{l}\text { Duration of follow up, mean } \\
\quad \pm \mathrm{SD} \text { (months) }\end{array}$ & $74.1 \pm 37.6$ & $55.5 \pm 27.2$ & $90.2 \pm 31.8$ & $0.001 *$ \\
\hline
\end{tabular}

HBV, hepatitis B virus; HCC, hepatocellular carcinoma; AVT, antiviral therapy; AFP, alpha-fetoprotein; MELD, model for end-stage liver disease; $\mathrm{HBeAg}$, hepatitis B E antigen. Continuous variables reported as median (range). Categorical variables reported as $n(\%) .{ }^{+}$Low barrier antiviral therapy included nucleos(t)ide analogues such as lamivudine, telbivudine, and adefovir. ${ }^{\ddagger}$ High barrier antiviral therapy included nucleos(t)ide analogues such as entecavir and tenofovir.; ${ }^{*} p<0.005$.

Baseline and follow-up values of the model for end-stage liver disease (MELD) score and serum biomarkers of liver fibrosis are compared in Table 2. Baseline values were calculated using biochemical variables measured at the time of the surgery and follow-up values were calculated using the results obtained at the time of the index follow-up LSM assessment. 
Table 2. Changes in noninvasive parameters of fibrosis during follow-up.

\begin{tabular}{cccc}
\hline Variables & Baseline & Follow Up & $p$-Value \\
\hline MELD ${ }^{\dagger}$ & & & \\
No recurrence & $16.2 \pm 2.0$ & $16.0 \pm 1.9$ & 0.352 \\
Early recurrence & $16.4 \pm 1.6$ & $16.3 \pm 1.8$ & 0.861 \\
Late recurrence & $16.6 \pm 2.0$ & $16.1 \pm 2.0$ & $0.003^{*}$ \\
\hline LSM (kPa) ${ }^{\ddagger}$ & & & \\
No recurrence & $12.8 \pm 7.4$ & $9.1 \pm 5.6$ & $<0.001^{*}$ \\
Early recurrence & $12.9 \pm 6.5$ & $12.7 \pm 8.0$ & 0.866 \\
Late recurrence & $13.9 \pm 6.8$ & $11.8 \pm 12.7$ & $<0.001^{*}$ \\
\hline Interquartile range $(\mathrm{kPa})$ & & & \\
No recurrence & $2.1 \pm 2.1$ & $1.2 \pm 1.1$ & $<0.001^{*}$ \\
Early recurrence & $1.6 \pm 1.4$ & $1.9 \pm 1.8$ & 0.635 \\
Late recurrence & $1.9 \pm 1.6$ & $1.6 \pm 1.8$ & $0.043^{*}$ \\
\hline APRI & & & 0.639 \\
No recurrence & $0.7 \pm 0.5$ & $0.7 \pm 0.6$ & $0.028^{*}$ \\
Early recurrence & $1.2 \pm 1.5$ & $0.7 \pm 0.9$ & $0.004^{*}$ \\
Late recurrence & $0.8 \pm 0.6$ & $0.6 \pm 0.5$ & 0.070 \\
\hline FIB-4 & & & 0.345 \\
No recurrence & $2.3 \pm 1.4$ & $2.4 \pm 1.2$ & $2.5 \pm 0.9$ \\
Early recurrence & $3.6 \pm 2.8$ & $2.6 \pm 1.5$ & \\
Late recurrence & $2.8 \pm 1.8$ & & \\
\hline
\end{tabular}

MELD, model for end-stage liver disease; LSM, liver stiffness measurement; APRI, aspartate aminotransferase-to-platelet ratio index; FIB-4, Fibrosis-4. Variables reported as mean \pm standard deviation. ${ }^{*} p<0.05 ;{ }^{\dagger}$ MELD, model for end-stage liver disease calculated as follows: MELD $=10 \times\left(\left(0.957 \times \log _{\mathrm{e}}(\mathrm{creatine})\right)+\right.$ $\left(0.378 \times \log _{\mathrm{e}}(\right.$ bilirubin $\left.)\right)+\left(1.12 \times \log _{\mathrm{e}}(\right.$ prothrombin time in international normalized ratio (INR)) $\left.)+6.43\right) ;{ }^{\ddagger} \mathrm{LSM}$ measured using transient elastography, expressed in kilopascal $(\mathrm{kPa})$.

Although all the patients had pathologically detected cirrhosis, advanced fibrosis was evident by APRI in $8.2 \%(15 / 184)$ and FIB-4 in $33.2 \%(61 / 184)$ of the patients at the time of liver resection. In addition, $\mathrm{LSM}>13 \mathrm{kPa}$, which would suggest liver cirrhosis, was shown in $38.3 \%(51 / 133)$, whereas LSM $>8 \mathrm{kPa}$, which is reported to predict an increased risk of HCC development [12], was demonstrated in $82.7 \%(110 / 133)$ of the patients at baseline (Figure 1). Follow-up assessments after receiving antiviral therapy for more than 12 months demonstrated no significant changes in the proportion of advanced liver fibrosis by APRI $(7.6 \%, 14 / 184)$ or FIB-4 $(35.3 \%, 65 / 184)(p=1.000, p=0.742$, respectively), whereas the proportion of high LSM $(>8 \mathrm{kPa})$ significantly decreased $(46.7 \%, 86 / 184)$ compared with that from the initial evaluation $(p<0.001)$ (Figure 1).

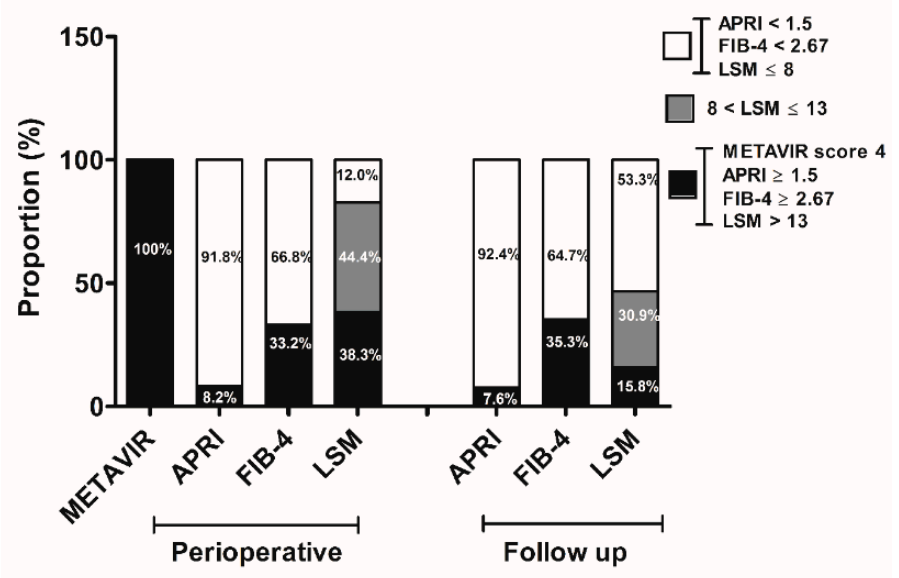

Figure 1. Changes in fibrotic burden. The proportion of patients with advanced fibrosis was assessed noninvasively by obtaining aspartate aminotransferase (AST)-platelet ratio index (APRI) and FIB-4 as well as liver stiffness (LS) values at the time of liver resection and during follow-up. METAVIR fibrosis score was evaluated from the resected liver at the time of HCC surgery. 


\subsection{Follow-Up LSM as a Predictor of HCC Recurrence}

The cumulative HCC recurrence-free survival rates (RFS) were $90.2 \%, 81.8 \%, 76.4 \%$, and $66.8 \%$ at $1,2,3$, and 5 years, respectively.

Late recurrence ( $>12$ months) was detected in 58 patients $(58 / 184,31.5 \%)$. Older age at liver resection ( $\geq 45$ year) (HR, 3.270; 95\% CI: 1.296-8.251; $p=0.012)$ and multiple HCC nodules (HR, 3.146; $95 \%$ CI: 1.396-7.089; $p=0.006$ ) suggested an increased risk of late recurrence (Table 3).

Table 3. Independent predictors of late recurrence after curative HCC resection in patients with pathologically confirmed liver cirrhosis under antiviral therapy.

\begin{tabular}{|c|c|c|c|}
\hline \multicolumn{2}{|l|}{ Univariate Analysis } & \multicolumn{2}{|c|}{ Multivariate Analysis } \\
\hline & $p$ & $\begin{array}{c}\text { Hazard Ratio (95\% } \\
\text { Confidence Interval (CI)) }\end{array}$ & $p$ \\
\hline $\begin{array}{l}\text { Age at resection } \\
\quad<45 \text { year } \\
\geq 45 \text { year }\end{array}$ & $0.003 *$ & $3.270(1.296-8.251)$ & $0.012 * *$ \\
\hline Gender & 0.526 & & \\
\hline $\begin{array}{l}\text { Operation type } \\
\text { Wedge resection } \\
\text { Segmentectomy } \\
\text { Lobectomy }\end{array}$ & 0.158 & & \\
\hline $\begin{array}{l}\text { Tumor size } \\
\leq 3 \mathrm{~cm} \\
>3 \mathrm{~cm}\end{array}$ & 0.415 & & \\
\hline $\begin{array}{l}\text { Tumor number } \\
\text { Single } \\
\text { Multiple }\end{array}$ & $0.001 *$ & $3.146(1.396-7.089)$ & $0.006^{* *}$ \\
\hline Microvascular invasion & 0.313 & & \\
\hline $\begin{array}{l}\text { Edmonson grade } \\
\qquad 2 \\
>2\end{array}$ & 0.628 & & \\
\hline $\begin{array}{l}\text { LSM, initial (kpa) } \\
\leq 8 \\
>8\end{array}$ & 0.644 & & \\
\hline $\begin{array}{l}\text { APRI, initial } \\
\quad<1.5 \\
\geq 1.5\end{array}$ & 0.366 & & \\
\hline $\begin{array}{l}\text { FIB-4, initial } \\
\quad<2.67 \\
\geq 2.67\end{array}$ & 0.176 & & \\
\hline $\begin{array}{l}\text { LSM, follow up }(\mathrm{kPa}) \\
\qquad 8 \\
>8\end{array}$ & $0.013 *$ & 0.519 (0.307-0.877) & $0.014^{* *}$ \\
\hline $\begin{array}{l}\text { APRI, follow up } \\
<1.5 \\
\geq 1.5\end{array}$ & 0.378 & & \\
\hline $\begin{array}{c}\text { FIB-4, follow up } \\
<2.67 \\
\geq 2.67\end{array}$ & 0.725 & & \\
\hline $\begin{array}{l}\text { AFP, initial } \\
<200 \mathrm{ng} / \mathrm{mL} \\
\geq 200 \mathrm{ng} / \mathrm{mL}\end{array}$ & 0.182 & & \\
\hline
\end{tabular}


Table 3. Cont.

\begin{tabular}{|c|c|c|c|}
\hline \multicolumn{2}{|l|}{ Univariate Analysis } & \multicolumn{2}{|c|}{ Multivariate Analysis } \\
\hline & $p$ & $\begin{array}{c}\text { Hazard Ratio (95\% } \\
\text { Confidence Interval (CI)) }\end{array}$ & $p$ \\
\hline $\begin{array}{l}\text { HBV DNA, initial } \\
<2000 \mathrm{IU} / \mathrm{mL} \\
\geq 2000 \mathrm{IU} / \mathrm{mL}\end{array}$ & 0.568 & & \\
\hline HBeAg positivity & 0.593 & & \\
\hline $\begin{array}{c}\text { Type of antiviral therapy }{ }^{\dagger} \\
\text { Low barrier } \\
\text { High barrier }\end{array}$ & 0.922 & & \\
\hline MELD, initial & 0.773 & & \\
\hline MELD, follow up & 0.425 & & \\
\hline
\end{tabular}

HCC, hepatocellular carcinoma; AVT, antiviral therapy; AFP, alpha-fetoprotein; LSM, liver stiffness measurement; APRI, aspartate aminotransferase-to-platelet ratio index; FIB-4, Fibrosis-4; MELD, model for end-stage liver disease. The initial values were assessed at the time of HCC resection and follow-up values were evaluated using the measurements obtained within 1 month before or after the last LS assessment. ${ }^{*} p<0.1,{ }^{* *} p<0.05,{ }^{\dagger}$ Type of antiviral therapy was categorized as low-barrier nucleos $(\mathrm{t})$ ide analogues such as lamivudine, telbivudine and adefovir, and high-barrier nucleos $(\mathrm{t})$ ide analogues such as entecavir and tenofovir. Please define "a" in the table, and cite ${ }^{\dagger}$.

Although all the patients were under antiviral therapy, achieving low fibrotic burden $(\mathrm{LSM} \leq 8 \mathrm{kPa})$ after antiviral therapy predicted reduced late recurrence and increased recurrence-free survival rates, which was pathologically confirmed at the time of liver resection $(p=0.013)$ (Figure 2A). Patients with LSM $>13 \mathrm{kPa}$ and suggested to have liver cirrhosis by LS criteria demonstrated RFS rates similar to those of patients with LSM under $13 \mathrm{kPa}$ but greater than $8 \mathrm{kPa}(p=0.936)$ (Figure 2B). However, the RFS rates of these patients were significantly lower than those of patients with $\mathrm{LSM} \leq 8 \mathrm{kPa}(p=0.047)$.

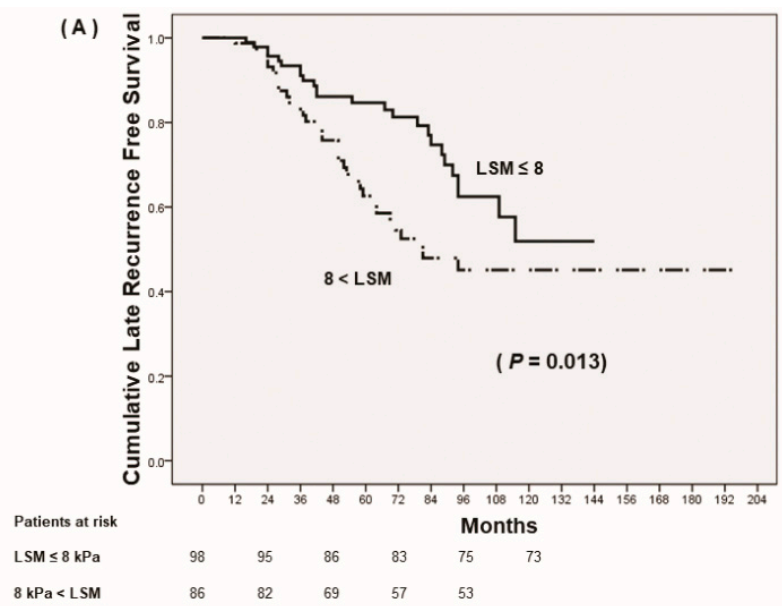

Figure 2. Cont. 

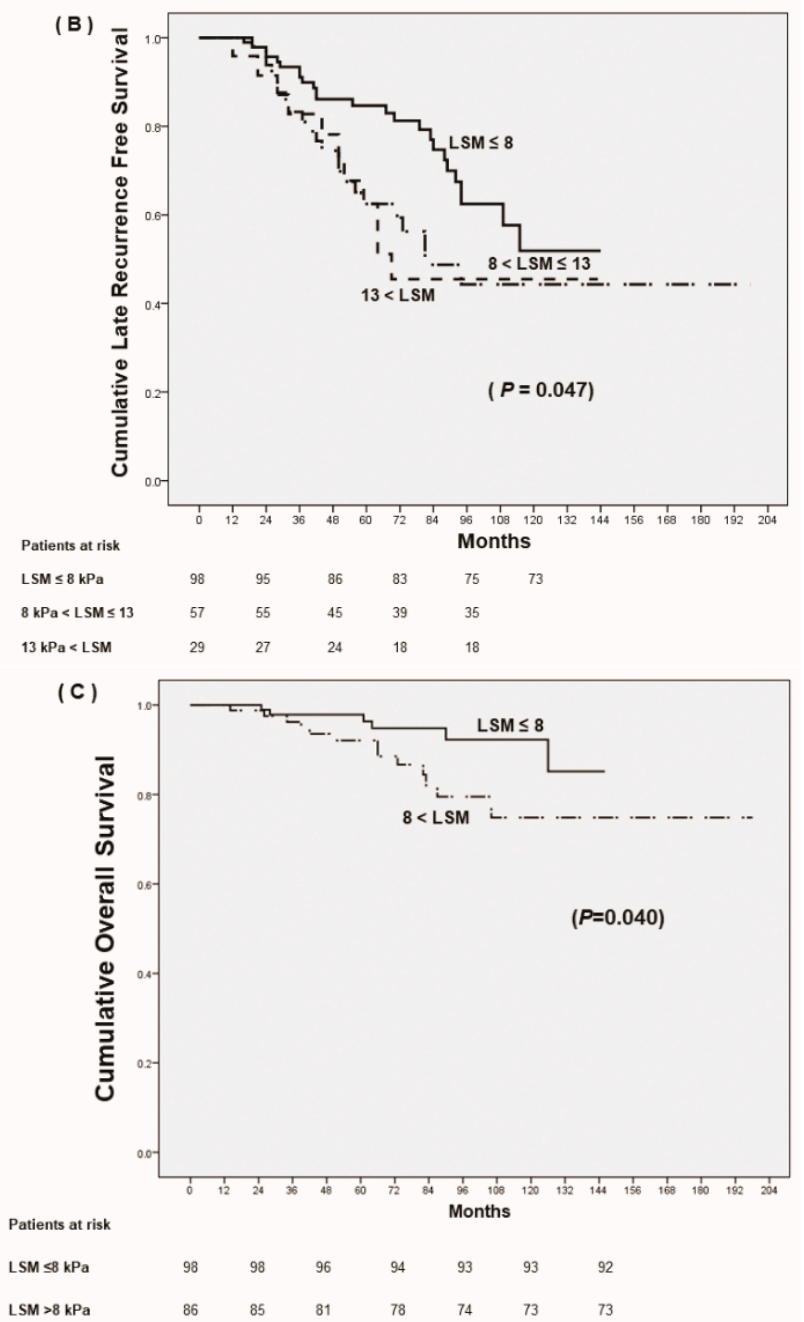

Figure 2. Prognosis of HCC after curative resection according to follow up-liver stiffness measurements (LSM). (A) Cumulative late recurrence-free survival based on stratified LSM, $\leq 8 \mathrm{kPa}$ vs. $>8 \mathrm{kPa}$. (B) Cumulative late recurrence-free survival based on stratified LSM, $\leq 8 \mathrm{kPa}$ vs. $8 \mathrm{kPa}<\mathrm{LSM} \leq 13 \mathrm{kPa}$ vs. $>13 \mathrm{kPa}$. (C) Cumulative overall survival.

Early recurrence ( $\leq 12$ months) was observed in 13 patients $(13 / 184,7.1 \%)$. Multiple HCC nodules (HR, 6.010; 95\% CI: 1.809-19.971; $p=0.003)$ and having microvascular invasion (HR, 2.779; 95\% CI: $1.126-6.856 ; p=0.027)$ suggested an increased risk of early recurrence, whereas preoperative low APRI score (HR, $0.169 ; 95 \%$ CI: $0.058-0.489 ; p=0.001)$ predicted a lower risk of early recurrence (Table 5). Having low LSMs $(\leq 8 \mathrm{kPa})$ during the follow-up period was not associated with early recurrence.

Table 4. Independent predictions of early recurrence after curative HCC resection in patients with pathologically confirmed liver cirrhosis under antiviral therapy.

\begin{tabular}{cccc}
\hline Univariate Analysis & \multicolumn{2}{c}{ Multivariate Analysis } \\
\hline & $p$ & Hazard Ratio (95\% CI) & $p$ \\
\hline $\begin{array}{c}\text { Age at resection } \\
<45 \text { year } \\
\geq 45 \text { years } \\
\text { Gender }\end{array}$ & 0.420 & \\
\hline
\end{tabular}


Table 5. Independent predictions of early recurrence after curative HCC resection in patients with pathologically confirmed liver cirrhosis under antiviral therapy.

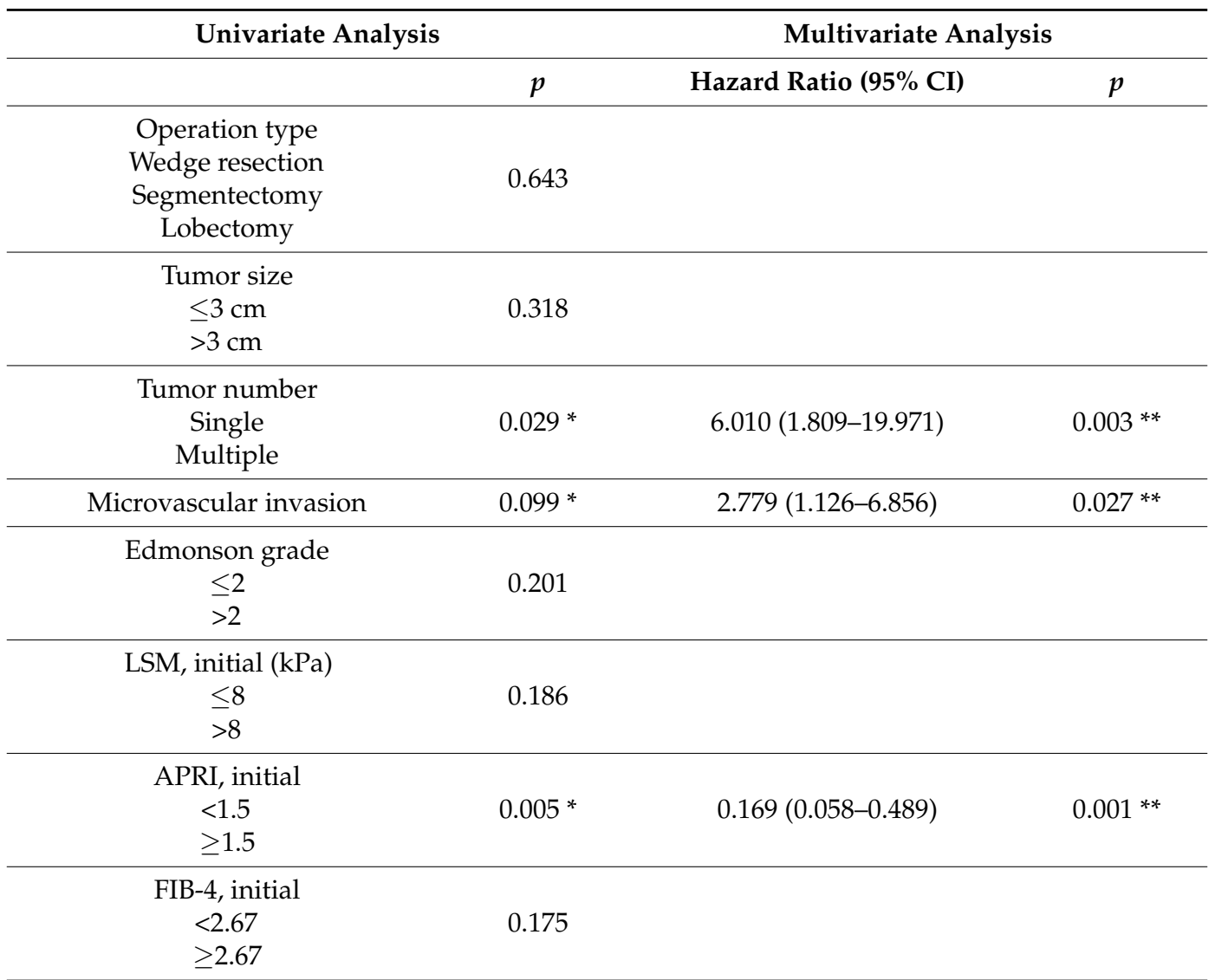

LSM, follow up $(\mathrm{kPa})$

$\leq 8 \quad 0.432$

$>8$

\begin{tabular}{|c|c|}
\hline $\begin{array}{l}\text { APRI, follow-up } \\
\quad<1.5 \\
\geq 1.5\end{array}$ & 0.942 \\
\hline $\begin{array}{l}\text { FIB-4, follow-up } \\
<2.67 \\
\geq 2.67\end{array}$ & 0.714 \\
\hline $\begin{array}{l}\text { AFP, initial } \\
<200 \mathrm{ng} / \mathrm{mL} \\
\geq 200 \mathrm{ng} / \mathrm{mL}\end{array}$ & 0.263 \\
\hline $\begin{array}{l}\text { HBV DNA, initial } \\
<2000 \mathrm{IU} / \mathrm{mL} \\
\geq 2000 \mathrm{IU} / \mathrm{mL}\end{array}$ & 0.163 \\
\hline HBeAg positivity & 0.466 \\
\hline $\begin{array}{c}\text { Type of antiviral therapy }{ }^{+} \\
\text {Low barrier } \\
\text { High barrier }\end{array}$ & 0.181 \\
\hline MELD, initial & 0.712 \\
\hline MELD, follow-up & 0.908 \\
\hline
\end{tabular}

HCC, hepatocellular carcinoma; AVT, antiviral therapy; AFP, alpha-fetoprotein; LSM, liver stiffness measurement; APRI, aspartate aminotransferase-to-platelet ratio index; FIB-4, Fibrosis-4; MELD, model for end-stage liver disease. The initial values were assessed at the time of HCC resection and follow up values were evaluated using the measurements obtained within 1 month before or after the last LS assessment. ${ }^{*} p<0.1{ }^{* *} p<0.05 ;{ }^{\dagger}$ Type of antiviral therapy was categorized as low-barrier nucleos $(\mathrm{t})$ ide analogues such as lamivudine, telbivudine and adefovir, and high-barrier nucleos(t)ide analogues such as entecavir and tenofovir. 


\subsection{Follow-up LSM as a Predictor of Mortality}

Among the patients with HBV-related HCC at BCLC 0-A with advanced fibrosis that underwent curative liver resection, the 1-, 2-, 3-, 5- year overall survival rates were $99.5 \%, 95.1 \%, 90.6 \%$ and $69.9 \%$, respectively. The multivariate regression analysis for determining independent factors for mortality is shown in Table 6. Although patients had pathologically confirmed liver cirrhosis at the time of HCC occurrence, achieving low LSM ( $\leq 8 \mathrm{kPa})$ after antiviral therapy suggested decreased cumulative incidence of mortality $(p=0.040)$ (Figure 2C). In addition, not having microvascular invasion of tumor, without early tumor recurrence ( $\leq 12$ months), and low APRI score at the time of tumor resection suggested decreased mortality.

Table 6. Independent predictions of mortality after curative HCC resection in patients with pathologically confirmed liver cirrhosis under antiviral therapy.

\begin{tabular}{|c|c|c|c|}
\hline \multicolumn{2}{|l|}{ Univariate Analysis } & \multicolumn{2}{|c|}{ Multivariate Analysis } \\
\hline & $p$ & Hazard Ratio (95\% CI) & $p$ \\
\hline $\begin{array}{c}\text { Age at resection } \\
<45 \text { year } \\
\geq 45 \text { years }\end{array}$ & 0.014 * & $1.76 \times 10^{5}\left(0.00-2.235 \times 10^{194}\right)$ & 0.957 \\
\hline Gender & 0.039 * & $0.00\left(0.00-3.643 \times 10^{187}\right)$ & 0.957 \\
\hline $\begin{array}{l}\text { Operation type } \\
\text { Wedge resection } \\
\text { Segmentectomy } \\
\text { Lobectomy }\end{array}$ & 0.112 & & \\
\hline $\begin{array}{l}\text { Tumor size } \\
\begin{array}{l}\leq 3 \mathrm{~cm} \\
>3 \mathrm{~cm}\end{array}\end{array}$ & 0.183 & & \\
\hline $\begin{array}{c}\text { Tumor number } \\
\text { Single } \\
\text { Multiple }\end{array}$ & 0.273 & & \\
\hline Microvascular invasion & $0.039 *$ & $3.191(1.188-8.568)$ & $0.021 * *$ \\
\hline $\begin{array}{c}\text { Edmonson grade } \\
\leq 2 \\
>2\end{array}$ & 0.384 & & \\
\hline Early Recurrence ( $\leq 12$ months) & $0.000 *$ & $9.416(3.566-24.861)$ & $0.000 * *$ \\
\hline Late Recurrence (>12 months) & $0.000 *$ & $3.366(0.605-18.724)$ & 0.119 \\
\hline $\begin{array}{l}\mathrm{LSM}^{*} \text { initial }(\mathrm{kPa}) \\
\leq 8 \\
>8\end{array}$ & 0.172 & & \\
\hline $\begin{array}{l}\text { LSM, follow-up }(\mathrm{kPa}) \\
\qquad \begin{array}{c}\leq 8 \\
>8\end{array}\end{array}$ & $0.040 *$ & $0.364(0.136-0.976)$ & $0.045^{* *}$ \\
\hline $\begin{array}{l}\text { APRI, initial } \\
\quad<1.5 \\
\quad \geq 1.5\end{array}$ & $0.002 *$ & $0.251(0.086-0.759)$ & $0.014^{* *}$ \\
\hline $\begin{array}{c}\text { APRI, follow-up } \\
<1.5 \\
\geq 1.5\end{array}$ & 0.034 * & $0.341(0.097-1.203)$ & 0.095 \\
\hline $\begin{array}{l}\text { FIB-4, initial } \\
\quad<2.67 \\
\geq 2.67\end{array}$ & 0.047 * & $1.261(0.345-4.613)$ & 0.726 \\
\hline $\begin{array}{l}\text { FIB-4, follow-up } \\
\quad<2.67 \\
\geq 2.67\end{array}$ & 0.168 & & \\
\hline
\end{tabular}


Table 6. Cont.

\begin{tabular}{cccc}
\hline \multicolumn{1}{c}{ Univariate Analysis } & \multicolumn{2}{c}{ Multivariate Analysis } \\
& $p$ & Hazard Ratio (95\% CI) & $p$ \\
\hline $\begin{array}{c}\text { AFP, initial } \\
<200 \mathrm{ng} / \mathrm{mL} \\
\geq 200 \mathrm{ng} / \mathrm{mL}\end{array}$ & $0.093^{*}$ & $0.615(0.071-5.350)$ & 0.660 \\
\hline $\begin{array}{c}\text { HBV DNA, initial } \\
<2000 \mathrm{IU} / \mathrm{mL} \\
\geq 2000 \mathrm{IU} / \mathrm{mL}\end{array}$ & 0.588 & & \\
\hline HBeAg positivity & 0.348 & & \\
\hline $\begin{array}{c}\text { Type of antiviral therapy }{ }^{+} \\
\text {Low barrier }\end{array}$ & $0.095 *$ & $0.249(0.060-1.038)$ \\
\hline High barrier & & \\
\hline MELD, initial & 0.395 & \\
\hline MELD, follow-up & 0.932 & \\
\hline
\end{tabular}

HCC, hepatocellular carcinoma; AVT, antiviral therapy; AFP, alpha-fetoprotein; LSM, liver stiffness measurement; APRI, aspartate aminotransferase -platelet ratio index; FIB-4, Fibrosis-4; MELD, model for end-stage liver disease. The initial values were assessed at the time of HCC resection and follow-up values were evaluated using the measurements obtained within 1 month before or after the last LS assessment. ${ }^{*} p<0.1 ;{ }^{* *} p<0.005$; ${ }^{+}$Type of antiviral therapy was categorized as low-barrier nucleos(t)ide analogues such as lamivudine, telbivudine and adefovir, and high-barrier nucleos $(\mathrm{t})$ ide analogues such as entecavir and tenofovir.

\section{Discussion}

This study demonstrates that among HCC patients with pathologically diagnosed liver cirrhosis receiving under antiviral therapy after HCC resection, achieving a decreased LSM to the point where lower fibrotic burden is likely to be suggested was associated with a significantly reduced risk of late recurrence and had a beneficial effect on overall survival.

Liver cirrhosis has been recognized as the most powerful risk factor of HCC development [2,3]. Although liver cirrhosis was previously thought to be irreversible, evidence supporting the reversal of cirrhosis by eliminating the underlying cause of liver injury, such as by using antiviral therapy, has accumulated $[4-7,28]$. Whether the reversal of liver fibrosis by antiviral therapy would lead to a reduction in HCC occurrence, especially in those with liver cirrhosis, is still in dispute. A previous study reported that antiviral therapy failed to prevent HCC development in patients with cirrhosis [29], whereas more recent studies suggested that antiviral therapy reduced the risk of HCC occurrence even in patients with liver cirrhosis [30,31]. However, all these studied could not clearly explain whether the effect of antiviral therapy was associated with an improvement in liver fibrosis or with suppressed HBV replication. Moreover, it is not clearly delineated which patients would benefit with decreased tumor recurrence after antiviral therapy.

Sequential liver biopsy is very difficult to perform due to its invasiveness and related ethical problems. Instead, LSM by TE has been widely accepted to be well correlated with pathologic stages of liver fibrosis [32-34], and several studies investigated the value of LSM in predicting the risk of de novo HCC development $[12,14,27,34,35]$. These studies suggest that higher LSM predicted an increased risk of HCC development, and even though the cutoff values for the increased risk may still need to be settled, they were generally accepted to be $>8 \mathrm{kPa}$, indicating high probability of a fibrosis stage around F3. Recently, it has been reported that LSM can be dynamically changed in both HCV and HBV infection after viral suppression or eradication, and this might result in a decreased risk of HCC development and liver-related events $[18,20,21]$. However, it has not been evaluated whether a reduction in LSM by antiviral therapy in cirrhotic HCC patients would result in a decreased risk of HCC recurrence, especially at a later stage after curative HCC treatment. These patients already experienced HCC development in a fibrotic liver background, and there has not been a study showing that the mere reduction in LSM by antiviral therapy would be a meaningful marker predicting changes in HCC prognosis in these patients. In our study, the results suggest that among cirrhotic HCC patients 
under antiviral therapy after surgical HCC treatment, achieving LSM $\leq 8 \mathrm{kPa}$ following antiviral therapy indicated a significantly reduced risk of HCC late recurrence and might be a useful marker predicting de novo recurrence after the curative liver resection.

It has been suggested that HCC early recurrence probably represents primary metastasis from the initial tumor and is dependent on tumor factors such as tumor numbers, existence of vascular invasion, and resection margin $[23,36,37]$. As for late recurrence, it is likely to result from multi-centric occurrence and be associated with underlying liver disease such as high HBV DNA level, liver inflammation, and severity of liver fibrosis [38]. Although it can still be argued that reduced LSM $\leq 8 \mathrm{kPa}$ may not necessarily indicate the pathological reversal of liver cirrhosis, low LSM can still be a reliable marker predicting favorable oncologic outcomes in cirrhotic HCC patients.

Our study has several limitations, mainly due to its retrospective nature. First, durations of antiviral therapy varied among the patients. The response to antiviral therapy in suppressing viral replication and ameliorating liver histology depends on the duration of antiviral therapy. However, all but only one patient had HBV DNA > 2000 IU/mL and 156 patients $(156 / 184,84.8 \%$ ) had undetectable HBV DNA during follow-up, suggesting that HBV replication had been effectively controlled. Whether there is a relationship between duration of undetectable HBV DNA and dynamic changes of LSM has not been adequately investigated and was beyond the scope of this study. Second, being a retrospective observational study, the point of follow-up LSM evaluation was not identical for all patients, although they were all at least 12 months after HCC resection and antiviral therapy start. Third, although all the patients had pathologically detected liver cirrhosis, a follow-up biopsy could not be performed, and decreased fibrotic burden could be estimated by achieving LSM $\leq 8 \mathrm{kPa}$, which is suggestive of having a METAVIR fibrosis score $\leq 2$. However, it is not practical to have a liver biopsy just to evaluate liver cirrhosis regression, and achieving low LSM seems to be meaningful for predicting prognosis even in HCC patients with cirrhosis.

\section{Conclusions}

Our study suggests that low LSM ( $\leq 8 \mathrm{kPa})$ after antiviral therapy in HBV-related HCC patients with pathologically detected liver cirrhosis may be a good prognostic marker for predicting late HCC recurrence and overall survival after curative liver resection.

Author Contributions: Conceptualization, J.I.L. and H.W.L.; Data curation, J.I.L.; Formal analysis, J.I.L. and S.U.K.; Investigation, J.I.L. and S.U.K.; Methodology, S.H.A. and K.S.L.; Project administration, J.I.L.; Resources, J.I.L., S.U.K., S.H.A., K.S.L.; Funding acquisition, J.I.L.; Software, J.I.L.; Supervision, J.I.L. and S.H.A.; Validation, S.U.K.; Writing—original draft, J.I.L.; Writing—review \& editing, H.W.L.

Funding: This research was supported by Basic Science Research Program through the National Research Foundation of Korea (NRF) funded by the Ministry of Science, ICT \& Future Planning (NRF-2016R1A2B4015192).

Conflicts of Interest: The authors have nothing to disclose.

\section{References}

1. Yuen, M.F.; Tanaka, Y.; Shinkai, N.; Poon, R.T.; But, D.Y.; Fong, D.Y.; Fung, J.; Wong, D.K.; Yuen, J.C.; Mizokami, M.; et al. Risk for hepatocellular carcinoma with respect to hepatitis b virus genotypes b/c, specific mutations of enhancer ii/core promoter/precore regions and hbv DNA levels. Gut 2008, 57, 98-102. [CrossRef] [PubMed]

2. Sasaki, Y.; Imaoka, S.; Masutani, S.; Ohashi, I.; Ishikawa, O.; Koyama, H.; Iwanaga, T. Influence of coexisting cirrhosis on long-term prognosis after surgery in patients with hepatocellular-carcinoma. Surgery 1992, 112, 515-521. [PubMed]

3. Poon, R.T.P.; Fan, S.T.; Wong, J. Risk factors, prevention, and management of postoperative recurrence after resection of hepatocellular carcinoma. Ann. Surg. 2000, 232, 10-24. [CrossRef]

4. Dienstag, J.L.; Goldin, R.D.; Heathcote, E.J.; Hann, H.W.L.; Woessner, M.; Stephenson, S.L.; Gardner, S.; Gray, D.F.; Schiff, E.R. Histological outcome during long-term lamivudine therapy. Gastroenterology 2003, 124, 105-117. [CrossRef] 
5. Liaw, Y.F.; Sung, J.J.Y.; Chow, W.C.; Farrell, G.; Lee, C.Z.; Yuen, H.; Tanwandee, T.; Tao, Q.M.; Shue, K.; Keene, O.N.; et al. Lamivudine for patients with chronic hepatitis $\mathrm{b}$ and advanced liver disease. N. Engl. J. Med. 2004, 351, 1521-1531. [CrossRef]

6. Chang, T.T.; Liaw, Y.F.; Wu, S.S.; Schiff, E.; Han, K.H.; Lai, C.L.; Safadi, R.; Lee, S.S.; Halota, W.; Goodman, Z.; et al. Long-term entecavir therapy results in the reversal of fibrosis/cirrhosis and continued histological improvement in patients with chronic hepatitis b. Hepatology 2010, 52, 886-893. [CrossRef]

7. Marcellin, P.; Gane, E.; Buti, M.; Afdhal, N.; Sievert, W.; Jacobson, I.M.; Washington, M.K.; Germanidis, G.; Flaherty, J.F.; Schall, R.A.; et al. Regression of cirrhosis during treatment with tenofovir disoproxil fumarate for chronic hepatitis b: A 5-year open-label follow-up study. Lancet 2013, 381, 468-475. [CrossRef]

8. Spinzi, G.; Terruzzi, V.; Minoli, G. Liver biopsy. N. Engl. J. Med. 2001, 344, 2030.

9. Marcellin, P.; Ziol, M.; Bedossa, P.; Douvin, C.; Poupon, R.; de Ledinghen, V.; Beaugrand, M. Non-invasive assessment of liver fibrosis by stiffness measurement in patients with chronic hepatitis b. Liver Int. 2009, 29, 242-247. [CrossRef] [PubMed]

10. Verveer, C.; Zondervan, P.E.; ten Kate, F.J.; Hansen, B.E.; Janssen, H.L.; de Knegt, R.J. Evaluation of transient elastography for fibrosis assessment compared with large biopsies in chronic hepatitis B and C. Liver Int. 2012, 32, 622-628. [CrossRef]

11. Li, Y.; Huang, Y.S.; Wang, Z.Z.; Yang, Z.R.; Sun, F.; Zhan, S.Y.; Liu, X.E.; Zhuang, H. Systematic review with meta-analysis: The diagnostic accuracy of transient elastography for the staging of liver fibrosis in patients with chronic hepatitis b. Aliment. Pharmacol. Ther. 2016, 43, 458-469. [CrossRef]

12. Jung, K.S.; Kim, S.U.; Ahn, S.H.; Park, Y.N.; Kim, D.Y.; Park, J.Y.; Chon, C.Y.; Choi, E.H.; Han, K.H. Risk assessment of hepatitis b virus-related hepatocellular carcinoma development using liver stiffness measurement (fibroscan). Hepatology 2011, 53, 885-894.

13. Kim, M.N.; Kim, B.K.; Park, J.Y.; Kim, D.Y.; Ahn, S.H.; Han, K.H.; Kim, S.U. Increased risk of developing hepatocellular carcinoma in chronic hepatitis b patients with transient elastography-defined subclinical cirrhosis. Hepatology 2014, 60, 978a-979a.

14. Shin, S.H.; Kim, S.U.; Park, J.Y.; Kim, D.Y.; Ahn, S.H.; Han, K.H.; Kim, B.K. Liver stiffness-based model for prediction of hepatocellular carcinoma in chronic hepatitis b virus infection: Comparison with histological fibrosis. Liver Int. 2015, 35, 1054-1062. [CrossRef]

15. Kuo, Y.H.; Lu, S.N.; Chen, C.H.; Chang, K.C.; Hung, C.H.; Tai, W.C.; Tsai, M.C.; Tseng, P.L.; Hu, T.H.; Wang, J.H. The changes of liver stiffness and its associated factors for chronic hepatitis $b$ patients with entecavir therapy. PLoS ONE 2014, 9, e93160. [CrossRef] [PubMed]

16. Kim, M.N.; Kim, S.U.; Kim, B.K.; Park, J.Y.; Kim, D.Y.; Ahn, S.H.; Han, K.H. Long-term changes of liver stiffness values assessed using transient elastography in patients with chronic hepatitis $\mathrm{b}$ receiving entecavir. Liver Int. 2014, 34, 1216-1223. [CrossRef] [PubMed]

17. Chon, Y.E.; Park, J.Y.; Myoung, S.M.; Jung, K.S.; Kim, B.K.; Kim, S.U.; Kim, D.Y.; Ahn, S.H.; Han, K.H. Improvement of liver fibrosis after long-term antiviral therapy assessed by fibroscan in chronic hepatitis $\mathrm{b}$ patients with advanced fibrosis. Am. J. Gastroenterol. 2017, 112, 882-891. [CrossRef]

18. Kim, B.S.; Seo, Y.S.; Kim, Y.S.; Lee, C.H.; Lee, H.A.; Um, S.H.; Yoo, J.J.; Kim, S.G.; Suh, S.J.; Jung, Y.K.; et al. Reduced risk of hepatocellular carcinoma by achieving a subcirrhotic liver stiffness through antiviral agents in hepatitis b virus-related advanced fibrosis or cirrhosis. J. Gastroenterol. Hepatol. 2018, 33, 503-510. [CrossRef] [PubMed]

19. Sasaki, K.; Shindoh, J.; Margonis, G.A.; Nishioka, Y.; Andreatos, N.; Sekine, A.; Hashimoto, M.; Pawlik, T.M. Effect of background liver cirrhosis on outcomes of hepatectomy for hepatocellular carcinoma. JAMA Surg. 2017, 152, e165059. [CrossRef]

20. Wu, S.S.; Kong, Y.Y.; Piao, H.X.; Jiang, W.; Xie, W.; Chen, Y.P.; Lu, L.G.; Ma, A.L.; Xie, S.B.; Ding, H.G.; et al. On-treatment changes of liver stiffness at week 26 could predict 2-year clinical outcomes in HBV-related compensated cirrhosis. Liver Int. 2018, 38, 1045-1054.

21. Singh, S.; Facciorusso, A.; Loomba, R.; Falck-Ytter, Y.T. Magnitude and kinetics of decrease in liver stiffness after antiviral therapy in patients with chronic hepatitis c: A systematic review and meta-analysis. Clin. Gastroenterol. Hepatol. 2018, 16, 27-38. [CrossRef] [PubMed]

22. D'Ambrosio, R.; Aghemo, A.; Fraquelli, M.; Rumi, M.G.; Donato, M.F.; Paradis, V.; Bedossa, P.; Colombo, M. The diagnostic accuracy of fibroscan for cirrhosis is influenced by liver morphometry in HCV patients with a sustained virological response. J. Hepatol. 2013, 59, 251-256. [CrossRef] 
23. Poon, R.T.; Fan, S.T.; Ng, I.O.; Wong, J. Significance of resection margin in hepatectomy for hepatocellular carcinoma: A critical reappraisal. Ann. Surg. 2000, 231, 544-551. [CrossRef]

24. Yamamoto, Y.; Ikoma, H.; Morimura, R.; Konishi, H.; Murayama, Y.; Komatsu, S.; Shiozaki, A.; Kuriu, Y.; Kubota, T.; Nakanishi, M.; et al. Optimal duration of the early and late recurrence of hepatocellular carcinoma after hepatectomy. World J. Gastroenterol. 2015, 21, 1207-1215. [CrossRef]

25. McPherson, S.; Stewart, S.F.; Henderson, E.; Burt, A.D.; Day, C.P. Simple non-invasive fibrosis scoring systems can reliably exclude advanced fibrosis in patients with non-alcoholic fatty liver disease. Gut 2010, 59, 1265-1269. [CrossRef]

26. Shah, A.G.; Lydecker, A.; Murray, K.; Tetri, B.N.; Contos, M.J.; Sanyal, A.J.; Nash Clinical Research Network. Comparison of noninvasive markers of fibrosis in patients with nonalcoholic fatty liver disease. Clin. Gastroenterol. Hepatol. 2009, 7, 1104-1112. [CrossRef]

27. Kim, M.N.; Kim, S.U.; Kim, B.K.; Park, J.Y.; Kim, D.Y.; Ahn, S.H.; Song, K.J.; Park, Y.N.; Han, K.H. Increased risk of hepatocellular carcinoma in chronic hepatitis B patients with transient elastography-defined subclinical cirrhosis. Hepatology 2015, 61, 1851-1859. [PubMed]

28. Schiff, E.R.; Lee, S.S.; Chao, Y.C.; Kew Yoon, S.; Bessone, F.; Wu, S.S.; Kryczka, W.; Lurie, Y.; Gadano, A.; Kitis, G.; et al. Long-term treatment with entecavir induces reversal of advanced fibrosis or cirrhosis in patients with chronic hepatitis b. Clin. Gastroenterol. Hepatol. 2011, 9, 274-276. [CrossRef]

29. Papatheodoridis, G.V.; Manolakopoulos, S.; Touloumi, G.; Vourli, G.; Raptopoulou-Gigi, M.; Vafiadis-Zoumbouli, I.; Vasiliadis, T.; Mimidis, K.; Gogos, C.; Ketikoglou, I.; et al. Virological suppression does not prevent the development of hepatocellular carcinoma in hbeag-negative chronic hepatitis b patients with cirrhosis receiving oral antiviral(s) starting with lamivudine monotherapy: Results of the nationwide hepnet. Greece cohort study. Gut 2011, 60, 1109-1116.

30. Su, T.H.; Hu, T.H.; Chen, C.Y.; Huang, Y.H.; Chuang, W.L.; Lin, C.C.; Wang, C.C.; Su, W.W.; Chen, M.Y.; Peng, C.Y.; et al. Four-year entecavir therapy reduces hepatocellular carcinoma, cirrhotic events and mortality in chronic hepatitis B patients. Liver Int. 2016, 36, 1755-1764. [CrossRef] [PubMed]

31. Zhang, P.; Liu, Q.; Yuan, M.; Wang, L. Tenofovir disoproxil fumarate reduce incidence of HCC development in CHB-patients with compensated cirrhosis. Infect. Agents Cancer 2018, 13, 30. [PubMed]

32. Branchi, F.; Conti, C.B.; Baccarin, A.; Lampertico, P.; Conte, D.; Frquelli, M. Non-invasive assessment of liver fibrosis in chronic hepatitis b. World J. Gastroenterol. 2014, 20, 14568-14580. [CrossRef] [PubMed]

33. Vigano, M.; Paggi, S.; Lampertico, P.; Fraquelli, M.; Massironi, S.; Ronchi, G.; Rigamonti, C.; Conte, D.; Colombo, M. Dual cut-off transient elastography to assess liver fibrosis in chronic hepatitis b: A cohort study with internal validation. Aliment. Pharmacol. Ther. 2011, 34, 353-362. [CrossRef] [PubMed]

34. Seo, Y.S.; Kim, M.Y.; Kim, S.U.; Hyun, B.S.; Jang, J.Y.; Lee, J.W.; Lee, J.I.; Suh, S.J.; Park, S.Y.; Park, H.; et al. Accuracy of transient elastography in assessing liver fibrosis in chronic viral hepatitis: A multicentre, retrospective study. Liver Int. 2015, 35, 2246-2255. [CrossRef]

35. Wang, J.H.; Yen, Y.H.; Yao, C.C.; Hung, C.H.; Chen, C.H.; Hu, T.H.; Lee, C.M.; Lu, S.N. Liver stiffness-based score in hepatoma risk assessment for chronic hepatitis c patients after successful antiviral therapy. Liver Int. 2016, 36, 1793-1799.

36. Yamamoto, J.; Kosuge, T.; Takayama, T.; Shimada, K.; Yamasaki, S.; Ozaki, H.; Yamaguchi, N.; Makuuchi, M. Recurrence of hepatocellular carcinoma after surgery. Br. J. Surg. 1996, 83, 1219-1222. [CrossRef]

37. Poon, R.T.; Fan, S.T.; Ng, I.O.; Lo, C.M.; Liu, C.L.; Wong, J. Different risk factors and prognosis for early and late intrahepatic recurrence after resection of hepatocellular carcinoma. Cancer 2000, 89, 500-507. [CrossRef]

38. Wu, J.C.; Huang, Y.H.; Chau, G.Y.; Su, C.W.; Lai, C.R.; Lee, P.C.; Huo, T.I.; Sheen, I.J.; Lee, S.D.; Lui, W.Y. Risk factors for early and late recurrence in hepatitis B-related hepatocellular carcinoma. J. Hepatol. 2009, 51, 890-897. [CrossRef]

(C) 2019 by the authors. Licensee MDPI, Basel, Switzerland. This article is an open access article distributed under the terms and conditions of the Creative Commons Attribution (CC BY) license (http:/ / creativecommons.org/licenses/by/4.0/). 